\title{
Dispersion measures as immunization risk measures
}

\author{
Alejandro Balbás ${ }^{a, 1}$, Alfredo Ibáñez ${ }^{\text {b,* }}$, Susana López ${ }^{a, 1}$ \\ a Departamento de Economía de la Empresa, Universidad Carlos III de Madrid, cl Madrid, \\ 126, 28903 Getafe Madrid, Spain \\ ${ }^{\mathrm{b}}$ Departamento de Administración, Instituto Tecnológico Autónomo de México, ITAM, \\ Río Hondo N. 1, Col Tizapán-San Angel, 01000 México, DF, Mexico
}

Received 5 November 1998; accepted 28 December 2000

\begin{abstract}
The quadratic and linear cash flow dispersion measures $M^{2}$ and $\tilde{N}$ are two immunization risk measures designed to build immunized bond portfolios. This paper generalizes these two measures by showing that any dispersion measure is an immunization risk measure and therefore, it sets up a tool to be used in empirical testing. Each new measure is derived from a different set of shocks (changes on the term structure of interest rates) and depends on the corresponding subset of worst shocks. Consequently, a criterion for choosing appropriate immunization risk measures is to take those developed from the most reasonable sets of shocks and the associated subset of worst shocks and then select those that work best empirically. Adopting this approach, this paper then explores both numerical examples and a short empirical study on the Spanish Bond Market in the mid-1990s to show that measures between linear and quadratic are the most appropriate, and amongst them, the linear measure has the best properties. This confirms previous studies on US and Canadian markets that maturityconstrained-duration-matched portfolios also have good empirical behavior.
\end{abstract}

JEL classification: G11

Keywords: Immunization risk measures; Dispersion measures; Linear measure; Maturity-matching bonds; Twists on the term structure 


\section{Introduction}

The immunization risk measure $M^{2}$ was introduced by Fong and Vasicek (1984) to overcome the main limitation of the traditional theory of immunization as formalized by Fisher and Weil (1971). It is possible to assume specific changes on the term structure of interest rates and develop different duration measures. ${ }^{2}$ Unfortunately, these duration measures are valid and therefore immunization is achieved only if interest rate changes conform to the specific process assumed. Fong and Vasicek (1984) showed that if the derivative of the instantaneous forward interest rate changes has an upper bound, then the return of a matching duration portfolio is bound from below by the $M^{2}$ quadratic cash flow dispersion measure, which depends only on the structure of the portfolio. Therefore, minimizing the $M^{2}$ measure minimizes the immunization risk.

Recently, a new immunization risk measure $\tilde{N}$ was introduced by Balbás and Ibáñez (1998). This new measure is a linear cash flow dispersion measure and is developed by considering the general set of shocks in which the difference between any two shocks on the forward instantaneous interest rates has an upper bound. The duration matching portfolio that minimizes the $\tilde{N}$ measure also minimizes the immunization risk. Balbás and Ibáñez (1998) also show the tie between these immunization risk measures and what they call the "weak immunization condition". By minimizing either the $M^{2}$ or $\tilde{N}$ measure, the effect of the worst shock, which prevents immunization in each model, is minimized. ${ }^{3,4}$

This paper generalizes $M^{2}$ and $\tilde{N}$ by showing that any cash flow dispersion measure is an immunization risk measure and therefore, it sets up a tool to be used in empirical testing. Each new measure is derived from a different set of shocks (changes on the term structure of interest rates) and depends on the corresponding subset of worst shocks. Consequently, a criterion for choosing appropriate immunization risk measures is to take those developed from the most reasonable sets of shocks and the associated subset of worst shocks and then select those that work best empirically.

Empirical results support the use of $M^{2}$ and maturity matching bonds. For example, Lacey and Nawhalka (1993) empirically demonstrated the effectiveness of matching duration and minimizing the $M^{2}$ measure. Bierwag et al. (1993) showed that among matching duration portfolios, the inclusion of a maturity matching bond works best empirically. Balbás and Ibáñez (1998) illustrated with examples that the matching duration portfolio that minimizes the $\tilde{N}$ measure usually requires a matu-

\footnotetext{
${ }^{2}$ For instance, parallel shifts or other non-parallel shifts (Bierwag, 1977; Chambers et al., 1988; Prisman and Shores, 1988), shifts in an equilibrium models context (Cox et al., 1979; Brennan and Schwartz, 1983), or empirically estimated shifts (Litterman and Scheinkman, 1991; Chance and Jordan, 1996; and others). Furthermore, most of these strategies require no short selling constraints.

${ }^{3}$ Unless the trivial case that a zero coupon bond with appropriate maturity is available.

${ }^{4}$ Bowden (1997) and Barber and Copper (1998) also propose a new method to obtain the worst changes on the interest rates. However, neither approach is equivalent to the $M^{2}$ or $\tilde{N}$ methods.
} 
rity matching bond. Therefore, studying the relationship between dispersion measures and immunization risk measures is worthwhile.

Additionally, this paper presents an empirical study on the Spanish bond market for a brief period in the mid-1990s that tests the dispersion measures developed here against other standard immunization strategies from the literature. Among the dispersion measures, the empirical test and numerical examples show that the best immunization results are obtained by using measures between the linear and the quadratic, with the linear $\tilde{N}$ having the best properties. These tests also show that the linear measure usually includes a maturity matching bond. Furthermore, tests on maturity-constrained duration-matched portfolios, as suggested in Bierwag et al. (1993) and others, perform well empirically. This confirms previous empirical results on US and Canadian markets.

The paper is organized as follows. Section 2 derives a generalized measure of cash flow dispersion in a bond portfolio. Section 3 ranks these dispersion measures bearing in mind their associated set of shocks and worst shocks. Section 4 presents a short empirical study on the Spanish Bond Market. Section 5 concludes the paper.

\section{A generalized cash flow dispersion measure}

This section builds on Balbás and Ibáñez (1998). They developed a linear dispersion measure by considering the general set of shocks in which the difference between any two shocks on the forward instantaneous interest rates has an upper bound. This upper bound can be understood as a volatility measure in this immunization context. Further, they showed that within this set there exists a subset of "worst shocks", which affect any immunized portfolio most negatively. Finally, among this subset of worst shocks, there exists one element such that immunization is not feasible. The authors demonstrate that the portfolio that minimizes the linear risk measure is the portfolio that minimizes the effect of this one worst shock.

We are now in a position to introduce the main result of this paper. The following Theorem 1 extends Balbás and Ibáñez (1998) by introducing the general dispersion measure $M^{n}, n>0$. We refer the reader to Appendix A for the construction and proof of these results.

Let $[0, T]$ be the time interval with $t=0$ the present moment and $m$ be the investor planning period, $0<m<T$. Let us denote by $C$ the capital to invest, by $R$ the $m$ maturity zero coupon bond return, and by $K$ the set of feasible shocks on the term structure of instantaneous forward interest rates. Following the usual assumptions, portfolio $q$ pays a continuous coupon $c(t) \geqslant 0,0 \leqslant t \leqslant T$, whose current value is denoted by $c(t, 0)$. If $g(t), 0 \leqslant t \leqslant T$, represents the instantaneous forward interest rates and $k(t) \in K$ is a shock on $g(t)$, then the value of the $q$ portfolio at $m$, including the coupons paid before $m$, is denoted by $V(q, k)$.

We will consider an infinite number of possibilities for the convex set of feasible shocks, $K$, and for each one we will develop a dispersion measure. For arbitrary $n>1$, let us introduce the set 


$$
K_{n}=\left\{k(t) ; \frac{\mathrm{d} k(t)}{\mathrm{d} t} \leqslant \lambda(n-1)|t-m|^{n-2}, t \geqslant 0, t \neq m\right\} .
$$

We assume that the derivative is bounded in every set $K_{n}$ to prevent shocks for which $V(q, k)$ would vanish at $m$ since forward rates can fall before $m$ and increase after $m$. For example, when $n=2$, a feasible shock such as $k(t)=\lambda(t-m)$ is a simple polynomial of degree one and if this shock occurs, any coupon will lose all of its value at $m$ if the parameter $\lambda$ has no upper bounds. This is the Fong and Vasicek (1984) case where $\lambda$ is the maximum derivative that the shock can achieve. Note that all the above are convex sets.

Integrating (1) between $t$ and $m$, we can see all that feasible shocks obey the following relationship:

$$
\begin{array}{ll}
k(m)-k(t) \leqslant \lambda|t-m|^{n-1}, & 0 \leqslant t<m, \\
k(t)-k(m) \leqslant \lambda|t-m|^{n-1}, & m<t \leqslant T,
\end{array}
$$

where now we have bounded the variation of the shocks between instants $t$ and $m$.

For the case $n \leqslant 1$ let us consider the following set of shocks which bound the variation between two instants (greater and smaller than $m$ ), where $\lambda(\lambda>0)$ is a parameter on this bound:

$$
\begin{aligned}
K_{n}= & \left\{k(t) ; k\left(t_{2}\right)-k\left(t_{1}\right) \leqslant \lambda\left[\left(t_{2}-m\right)^{n-1}+\left(m-t_{1}\right)^{n-1}\right],\right. \\
& \left.0 \leqslant t_{1}<m<t_{2} \leqslant T\right\} .
\end{aligned}
$$

The case $n=1$ is a limit case. For $n \leqslant 1$, the derivative is not bounded as in (1), but the variation of the shocks between two instants (greater and smaller than $m$ ) is bounded as in (3). The shocks belonging to $K_{1}$ permit any derivative on the shocks, but bound the absolute variation of the shocks between any two instants by the parameter $2 \lambda .^{5}$

Theorem 1. For all $k \in K_{n}, n>0$, we have

$$
\frac{V(q, k)-R C}{R C} \geqslant \lambda_{0}(m-D)-\frac{\lambda}{n} M^{n}
$$

where $D$ is the Macaulay duration of the q portfolio, $\lambda_{0}$ depends on shock $k$ and is given by Lemma 2 in Appendix A, and $M^{n}$ is the dispersion measure,

$$
M^{n}=\int_{0}^{T} \frac{c(t, 0)}{C}|t-m|^{n} \mathrm{~d} t
$$

\footnotetext{
${ }^{5}$ The set $K=\left\{k(t) ;\left|k\left(t_{2}\right)-k\left(t_{1}\right)\right| \leqslant \lambda, 0 \leqslant t_{1}<m<t_{2} \leqslant T\right\}$ is quite close to the set considered in Balbás and Ibáñez (1998) and is included in $K_{1}$.
} 
Proof. See Appendix A.

If we now immunize against parallel shocks, $D=m$, we obtain

$$
\frac{V(q, k)-R C}{R C} \geqslant \frac{-\lambda}{n} M^{n}
$$

and therefore, any dispersion measure is an immunization risk measure. ${ }^{6}$

From the proof of Theorem 1, each dispersion measure is a weighting of how the worst shock with $\lambda_{0}=0$ impacts the interest rates with which each coupon has to be compounded. ${ }^{7}$ The weights equal the present value of the coupons. Minimizing a dispersion measure minimizes such effects. Now, if we consider this worst shock then immunization is not feasible, and we can see again the connection between the "weak immunization condition" and the strategies minimizing dispersion measures. Minimizing any dispersion measure minimizes the effect of the worst shock (for the associated set of shocks) that prevents immunization.

For the case $n \leqslant 0$, Appendix A shows that the sets $K_{n}^{*}$ contain worst shocks such that the effect of these shocks on interest rates between $t$ and $m$ is $-\infty$ for $t<m$ and $+\infty$ for $t>m$. Compounding this effect implies the value of any coupon at $m$ is zero. Therefore, although some dispersion measures are correct mathematically, they do not make economic sense since they are associated with unrealistic shocks and worst shocks. This fact will be used in the following section to choose among dispersion measures.

\section{Choosing a dispersion measure}

We have shown that each dispersion measure is derived from a different set of shocks and its corresponding subset of worst shocks. Consequently, we will consider the most appropriate dispersion measures to be those implied by the most reasonable sets of shocks and the associated subset of worst shocks. We will undertake this analysis by looking at each set of shocks (Eq. (1) for $n>1$ and Eq. (3) for $n \leqslant 1$ ) and worst shocks (Eq. (A.5) in Appendix A for all $n$ ).

A number of general dispersion measures, $M^{n}$, can be eliminated because they do not make economic sense. For example, Footnote 11 (in Appendix A) shows that for $n \leqslant 0$ the sets $K_{n}$ give rise to absurd worst shocks and risk immunization measures that are not dispersion measures. Therefore, they do not make economic sense. Eq. (A.5) demonstrates that dispersion measures with $0<n<1$ are also implied by unrealistic worst shocks. Furthermore, if twists on the term structure result from reasonable shocks, then (3) implies that these twists should not be allowed unless the parameter $\lambda$ is large enough. It is hard to explain why the parameter $\lambda$ should be

\footnotetext{
${ }^{6}$ Note that measure $M^{1}$ is denoted by $\tilde{N}$ in Balbás and Ibáñez (1998).

${ }^{7}$ Eq. (A.5) in Appendix A provides the general form of the subset of worst shocks, $K_{n}^{*}$, for all $n$.
} 
so large. Therefore, we do not consider measures such that $0<n<1$ to be suitable risk immunization measures. Finally, Eq. (A.5) shows that when $n>2$ the associated worst shocks are not reasonable as they are too large when $t$ is far from $m$. Therefore, the appropriate dispersion measures are those that are between the linear and the quadratic, i.e., $1 \leqslant n \leqslant 2$.

Balbás and Ibáñez (1998, pp. 1586-1587) made three arguments that are relevant to this discussion regarding the favorable properties of shocks in $K_{1}$ rather than in $K_{2}$. These arguments are also valid comparing $K_{1}$ with $K_{n}, 1<n<2$. Note however, as $n$ tends to 1 , the sets $K_{n}$ and $K_{1}$ become quite similar and the dispersion measures tend to the linear measure. Therefore, these arguments become weaker as $n$ tends to 1 and imply that measures close to the linear are developed from more reasonable shocks than those close to the quadratic one.

For $1<n<2$, the dispersion measures share properties of both the linear and quadratic dispersion measures. They do not constrain the derivative of the shock so much as the quadratic measure at $t$ close to $m$. On the other hand, they will permit more variations than the linear measure if $t$ is far from $m$. Both the linear and the quadratic measures do, however, share an advantage over the measures between them; the sets $K_{1}$ in (3) and $K_{2}$ in (1), from which these measures are deduced, do not depend on the parameter $m$.

To bring more light to the problem, we now present a simple example to see which portfolios minimize the dispersion measures. For simplicity, we consider an investor planning period of five years, $m=5$, and assume a flat term structure on the interest rates, $r=10 \%$.

Let us consider the set of coupon bonds presented in Table 1. The first column in Table 1 indicates the bond number, the second its maturity, the third its coupon (as a percentage), the fourth its coupon periodicity (in months), the fifth its bond duration (in years), and the last five columns correspond to its dispersion measures for $n=0.5,1,1.5,2$ and 2.5. In Table 1 we give the duration matching portfolios. The first column is the portfolio number, the second is the first bond in the portfolio, the third is the second bond in the portfolio, the fourth is the first bond percentage and the last five columns correspond to the dispersion measures given for $n=0.5,1,1.5,2$ and 2.5. The portfolios are arranged according to their $M^{1}$ measure.

Table 1

Set of bonds

\begin{tabular}{lllllllllc}
\hline $\begin{array}{l}\text { Bond } \\
\text { no. }\end{array}$ & $\begin{array}{l}\text { Maturity } \\
\text { (years) }\end{array}$ & $\begin{array}{l}\text { Coupon } \\
(\%)\end{array}$ & $\begin{array}{l}\text { Coupon } \\
\text { (monthly) }\end{array}$ & $\begin{array}{l}\text { Duration } \\
\text { (years) }\end{array}$ & $M^{0.5}$ & $M^{1}$ & $M^{1.5}$ & $M^{2}$ & $M^{2.5}$ \\
\hline 1 & 4 & 10 & 12 & 3.4823 & 1.1840 & 1.5176 & 2.1307 & 3.2713 & 5.4159 \\
2 & 5 & 10 & 12 & 4.1610 & 0.5043 & 0.8389 & 1.4539 & 2.5982 & 4.7497 \\
3 & 6 & 10 & 12 & 4.7759 & 1.1231 & 1.4588 & 2.0755 & 3.2231 & 5.3809 \\
4 & 7 & 10 & 12 & 5.3328 & 1.3555 & 2.0201 & 3.1025 & 4.9093 & 8.0007 \\
5 & 8 & 10 & 12 & 5.8368 & 1.5168 & 2.5281 & 4.3060 & 7.4515 & 13.051 \\
6 & 9 & 10 & 12 & 6.2928 & 1.6398 & 2.9878 & 5.5957 & 10.672 & 20.606 \\
\hline
\end{tabular}


Table 2

Matching-duration portfolios

\begin{tabular}{llllllllc}
\hline No. & 1st bond & 2nd bond & $\%(1 \mathrm{st})$ & $M^{0.5}$ & $M^{1}$ & $M^{1.5}$ & $M^{2}$ & $M^{2.5}$ \\
\hline 1 & 3 & 4 & 59.77 & 1.2166 & 1.6846132 & 2.4887 & 3.9014 & 6.4348 \\
2 & 3 & 5 & 78.88 & 1.2063 & 1.6846172 & 2.5465 & 4.1160 & 7.0007 \\
3 & 2 & 4 & 28.40 & 1.1137 & 1.6846198 & 2.6342 & 4.2528 & 7.0773 \\
4 & 3 & 6 & 85.23 & 1.1994 & 1.6846211 & 2.5954 & 4.3233 & 7.0773 \\
5 & 2 & 5 & 49.93 & 1.0112 & 1.6846342 & 2.8817 & 5.0279 & 8.9059 \\
6 & 2 & 6 & 60.64 & 0.9511 & 1.6846482 & 3.0839 & 5.7759 & 10.990 \\
7 & 1 & 4 & 17.98 & 1.3246 & 1.9297429 & 2.9277 & 4.6146 & 7.5358 \\
8 & 1 & 5 & 35.54 & 1.3985 & 2.1690124 & 3.5328 & 5.9657 & 10.3377 \\
9 & 1 & 6 & 46.00 & 1.4301 & 2.3115433 & 4.0018 & 7.2680 & 13.6187 \\
\hline
\end{tabular}

As Bierwag et al. (1993) showed, a bullet portfolio does not always minimize the $M^{2}$ measure. However, this simple example satisfies the conditions for a bullet portfolio to minimize the $M^{2}$ measure. We can see the smaller the $n$, the greater the possibility that the optimal portfolio includes a maturity matching bond (Bond 2). This is because the portfolios that include Bond 2 are closer to the optimal portfolio. As $n$ increases, there is a greater possibility that the optimal portfolio is a bullet portfolio. This example suggests the following interesting result. The linear measure, ceteris paribus, could be the border between the optimal portfolio including a maturity matching bond and a bullet portfolio. Measures lower than linear are minimized by portfolios that include a maturity matching bond; measures greater than linear by a bullet portfolio. Both portfolios minimize the $\tilde{N}$ linear measure. This result is not robust since, for instance, it depends on the convexity property noted in Bierwag et al. (1993), but it is illustrative of the linear measure.

Let us now focus on the $M^{2}$ and the $M^{1}$ measures and compare Portfolios 1 and 3 in Table 2. Although Bond 2 has the minimum $M^{2}$ and $M^{1}$ measures in Table 1, Portfolio 3 which includes Bond 2, minimizes the $M^{1}$ measure but has a high $M^{2}$ measure. An explanation for this could be as follows. Bond 2 in Portfolio 3 has a lower duration than Bond 3 in portfolio 1. Therefore, Portfolio 3 must invest more money in the second bond, Bond 4, to match the duration with $m$. Since $M^{2}$ is a quadratic dispersion measure, this second bond has a large impact on $M^{2}$, while the $M^{1}$ linear measure does not grow as fast in this second bond. Furthermore, the $M^{2}$ measure of Bond 2 is $80.61 \%$ of Bond 3. The $M^{1}$ measure of Bond 2, however, is only $57.51 \%$ of Bond 3. Consequently, Bond 2 is more appropriate to minimize $M^{1}$ and Bond 3 to minimize $M^{2}$.

\section{Empirical results on the Spanish Bond Market}

This section provides some empirical evidence for the theoretical results of Section 2 and the discussion in Section 3. We present a study of immunization strategies on 
the Spanish Bond Market in recent years. The evolution of interest rates in Spain has been marked by the process of convergence of this variable inside the framework of the European Union. In the early 1990s, Spain had higher rates than expected for the date of integration at 1999 and therefore during the study period (1993-1998) interest rates generally declined. For instance, at the beginning of 1993 the 3-year yieldto-maturity was around $12 \%$ while this rate equaled $4.5 \%$ at the end of 1998 , with the interest rates still falling. However, the period 1994-1995 saw a turn in this trend, and yields increased from approximately $8 \%$ to $11.5 \%$.

\subsection{The data}

Data on bid-ask bond prices are from the Bank of Spain. From these data, Navarro and Nave (1997) estimated a term structure of interest rates. We use this term structure to compute discount factors. ${ }^{8}$ Bond maturities range from 1,2 and 6 months to $1,2,3,4,5,6,10,14$ and 15 years. We have monthly data for the period from 16 April 1993 to 18 December 1995 . This corresponds to approximately 30 overlapping periods within which to conduct immunization strategies. However, given the importance of maturity matching $(\mathrm{m}-\mathrm{m})$ bonds in immunization strategies, we only consider 12 periods for which there is an m-m bond available. While 12 periods are insufficient for a complete empirical study, we believe that it is sufficient to demonstrate basic evidence of the theoretical results discussed earlier. We leave a more fundamental empirical study for further research. Given the recent period of study, we are also obligated to choose a planning period of $m=3$ years, shorter than the standard of 5 years. The bonds are all coupon bonds for average and long maturities, and the coupon is usually paid annually. The coupon reflects the level of interest rates, and bonds issued in 1993 have a coupon around $12 \%$, whereas bonds issued in 1995 have a coupon around 8\%.

\subsection{The tests}

We conduct 12 tests of periods ranging from 16 April 1993 to 18 December 1995 for a planning period of $m=3$ years. Each portfolio is rebalanced every time that one of the bonds in the portfolio pays a coupon. Given that the coupon is annual and that a portfolio contains two bonds, each portfolio is rebalanced approximately twice a year.

We consider three groups of strategies. In the first group, we test the strategies developed in Section 2. We test the matching-duration strategies that minimize the dispersion measures $M^{0.5}, M^{1}, M^{1.5}, M^{2}$ and $M^{2.5}$, a reasonable coverage of the spectrum of strategies for $0<n<\infty$.

\footnotetext{
${ }^{8}$ We are very grateful to Professors Navarro and Nave for providing us with several databases and comments regarding the empirical test.
} 
In the second group, we test the matching-duration strategies that include an m-m bond and that minimize the dispersion measures $M^{0.5}, M^{1}, M^{1.5}, M^{2}$ and $M^{2.5}$. In other words, we test portfolios that minimize these dispersion measures, but are constrained to matching-duration and the inclusion of a m-m bond. The purpose of the latter group is twofold, first to see if there is an improvement over the first group after including a m-m bond, and second, to see which of the first group strategies usually includes a m-m bond. Note that there are no theoretical arguments to include a m-m bond, only strong empirical evidence (see Bierwag et al., 1993).

The third group of strategies corresponds to a bullet, a maturity matching bullet (m-m-bu) and a maturity matching barbell (m-m-ba). These have been considered in prior studies, as in Bierwag et al. (1993), and again, we are interested in a comparison between these and group one strategies. Furthermore, in light of the example in Table 2, all of these almost minimize the $M^{1}$ measure, and therefore, we would also like to test whether they beat it.

As performance measures we have included the mean square error (MSE), and the frequency with which a strategy return was the closest to the promised one or target. We have also included the mean return for illustrative purposes. Given only 12 overlapping periods, we do not report statistical tests.

\subsection{The results}

Tables 3-5 contain the results of the empirical study. In Table 3, Group 1, the $M^{1}$ strategy has the best performance among all strategies. The $M^{0.5}$ strategy has the worst performance. Usually, the $M^{0.5}$ includes a m-m bond, but its poor performance reflects that it is derived from an unreasonable set of changes on interest rates. All of the measures greater than linear perform well and, in this respect, are nearly indistinguishable. Note that these strategies for 9 or 10 periods had the same outcome implying that the portfolio minimizing these measures at each rebalancing period was the same. Therefore, although they are derived from different sets of shocks, they could have similar empirical performance. This means that a very unreasonable measure, as derived from a large $n$, say $M^{100}$, could yield empirical performance as good as $M^{2}$.

In Table 4, Group 2, the best strategy is the $M^{1} \mathrm{~m}-\mathrm{m}$. The order of performance is the same as Table 3, and the overall performance is slightly worse than in Table 3, where portfolios were not constrained to include a m-m bond. If we compare columns from Tables 3 and 4 we note the following. Columns $M^{0.5}$ and $M^{0.5} \mathrm{~m}-\mathrm{m}$ are the same for each period. Therefore, the portfolio minimizing $M^{0.5}$ always includes a m-m bond at each rebalancing date. Columns $M^{1}$ and $M^{1} \mathrm{~m}-\mathrm{m}$ are the same for seven periods. This implies that for each rebalancing date in these periods, they were minimized by the same portfolio. Consequently, the portfolio minimizing $M^{1}$ almost always includes a $\mathrm{m}-\mathrm{m}$ bond. Measures greater than linear also can include a $\mathrm{m}-\mathrm{m}$ bond. ${ }^{9}$

\footnotetext{
${ }^{9}$ Perhaps, an $\mathrm{m}-\mathrm{m}$ bond is frequently included in the strategies because for each rebalancing date the duration of this bond is close to the planning period ( $\leqslant 3$ years), something that would probably fail for an initial planning period of 5 years.
} 
Table 3

Group 1 of strategies

\begin{tabular}{lllllll}
\hline Initial date & Target & $M^{0.5}$ & $M^{1}$ & $M^{1.5}$ & $M^{2}$ & $M^{2.5}$ \\
\hline 16.04 .93 & 39.057 & 38.641 & 38.921 & 38.860 & 38.852 & 38.852 \\
16.07 .93 & 32.099 & 31.991 & 31.897 & 31.893 & 31.893 & 31.893 \\
31.08 .93 & 28.179 & 27.988 & 28.000 & 28.000 & 28.000 & 28.000 \\
03.12 .93 & 26.932 & 26.520 & 26.804 & 26.780 & 26.780 & 26.828 \\
17.01 .94 & 23.870 & 24.197 & 23.850 & 23.808 & 23.808 & 23.824 \\
01.03 .94 & 25.376 & 25.505 & 25.261 & 25.323 & 25.323 & 25.323 \\
17.06 .94 & 32.060 & 31.829 & 32.059 & 32.049 & 32.048 & 32.048 \\
01.08 .94 & 30.682 & 30.552 & 30.655 & 30.730 & 30.735 & 30.735 \\
01.09 .95 & 34.997 & 34.634 & 35.000 & 35.081 & 35.081 & 35.081 \\
06.11 .95 & 33.452 & 33.403 & 33.491 & 33.472 & 33.472 & 33.472 \\
01.12 .95 & 31.862 & 31.808 & 31.861 & 31.870 & 31.870 & 31.870 \\
18.12 .95 & 30.284 & 30.228 & 30.349 & 30.353 & 30.353 & 30.353 \\
Mean return & 30.738 & 30.608 & 30.679 & 30.685 & 30.685 & 30.690 \\
Return closest & & $2 / 12$ & $7 / 12$ & $3 / 12$ & $3 / 12$ & $4 / 12$ \\
to target & & & & & & \\
MSE & & 0.0604 & 0.0106 & 0.0131 & 0.0135 & 0.0123 \\
\hline
\end{tabular}

Finally, Table 5, Group 3, shows that the portfolio with the best empirical behavior is $M^{1}$. The m-m-bu portfolio also performs well which confirms previous empirical results regarding the use of these strategies in US and Canadian markets. The bullet does not perform as well as expected and does not come close to the portfolio minimizing the $M^{2}$ measure. This is probably because the convexity property connecting both strategies in Bierwag et al. (1993) fails.

Table 4

Group 2 of strategies

\begin{tabular}{lllllll}
\hline Initial date & Target & $M^{0.5} \mathrm{~m}-\mathrm{m}$ & $M^{1} \mathrm{~m}-\mathrm{m}$ & $M^{1.5} \mathrm{~m}-\mathrm{m}$ & $M^{2} \mathrm{~m}-\mathrm{m}$ & $M^{2.5} \mathrm{~m}-\mathrm{m}$ \\
\hline 16.04 .93 & 39.057 & 38.641 & 38.921 & 38.860 & 38.852 & 38.852 \\
16.07 .93 & 32.099 & 31.991 & 31.917 & 31.915 & 31.915 & 31.915 \\
31.08 .93 & 28.179 & 27.988 & 28.027 & 28.022 & 28.004 & 28.004 \\
03.12 .93 & 26.932 & 26.520 & 26.804 & 26.780 & 26.780 & 26.780 \\
17.01 .94 & 23.870 & 24.197 & 23.946 & 23.754 & 23.734 & 23.734 \\
01.03 .94 & 25.376 & 25.505 & 25.261 & 25.323 & 25.323 & 25.323 \\
17.06 .94 & 32.060 & 31.829 & 31.958 & 31.970 & 31.970 & 31.970 \\
01.08 .94 & 30.682 & 30.552 & 30.655 & 30.730 & 30.735 & 30.735 \\
01.09 .95 & 34.997 & 34.634 & 35.047 & 35.016 & 35.024 & 35.016 \\
06.11 .95 & 33.452 & 33.403 & 33.491 & 33.469 & 33.469 & 33.469 \\
01.12 .95 & 31.862 & 31.808 & 31.886 & 31.875 & 31.875 & 31.875 \\
18.12 .95 & 30.284 & 30.228 & 30.349 & 30.353 & 30.353 & 30.353 \\
Mean return & 30.738 & 30.608 & 30.689 & 30.672 & 30.670 & 30.669 \\
Return closest & & $2 / 12$ & $5 / 12$ & $5 / 12$ & $4 / 12$ & $5 / 12$ \\
to target & & & & & & \\
MSE & & 0.0604 & 0.0108 & 0.0127 & 0.0140 & 0.0139 \\
\hline
\end{tabular}


Table 5

Group 3 of strategies

\begin{tabular}{lllllll}
\hline Initial date & Target & $M^{1}$ & $M^{1} \mathrm{~m}-\mathrm{m}$ & bullet & m-m-bu & m-m-ba \\
\hline 16.04 .93 & 39.057 & 38.921 & 38.921 & 38.699 & 38.786 & 38.750 \\
16.07 .93 & 32.099 & 31.897 & 31.917 & 32.018 & 31.898 & 31.980 \\
31.08 .93 & 28.179 & 28.000 & 28.027 & 27.999 & 28.005 & 27.955 \\
03.12 .93 & 26.932 & 26.804 & 26.804 & 26.738 & 26.779 & 26.493 \\
17.01 .94 & 23.870 & 23.850 & 23.946 & 23.530 & 23.742 & 24.167 \\
01.03 .94 & 25.376 & 25.261 & 25.261 & 25.240 & 25.363 & 25.466 \\
17.06 .94 & 32.060 & 32.059 & 31.958 & 32.028 & 31.953 & 31.751 \\
01.08 .94 & 30.682 & 30.655 & 30.655 & 30.688 & 30.688 & 30.558 \\
01.09 .95 & 34.997 & 35.000 & 35.047 & 35.148 & 35.050 & 34.613 \\
06.11 .95 & 33.452 & 33.491 & 33.491 & 33.465 & 33.471 & 33.288 \\
01.12 .95 & 31.862 & 31.861 & 31.886 & 31.875 & 31.879 & 31.722 \\
18.12 .95 & 30.284 & 30.349 & 30.349 & 30.365 & 30.345 & 30.188 \\
Mean return & 30.738 & 30.679 & 30.688 & 30.650 & 30.663 & 30.578 \\
Return closest & & $6 / 12$ & $3 / 12$ & $3 / 12$ & $3 / 12$ & $0 / 12$ \\
to target & & & & & & \\
MSE & & 0.0106 & 0.0108 & 0.0308 & 0.0169 & 0.0635 \\
\hline
\end{tabular}

The intuition developed in Section 3 generally agrees with the following conclusions for $m=3$ years:

1. The matching duration portfolio that minimizes the linear measure shows the best empirical performance. Furthermore, this portfolio usually includes a maturity matching bond.

2. Portfolios minimizing measures lower than linear (the $M^{0.5}$ measure) include a maturity matching bond, but they do not perform well empirically. These portfolios can be very different from those minimizing the linear measure.

3. Portfolios minimizing measures greater than the linear one perform well but not as well as the one minimizing the linear measure. This relatively good performance can be explained by the fact that these portfolios almost minimize the linear measure. The measure $M^{2.5}$, although not so appealing theoretically, performs well because the portfolio minimizing $M^{2.5}$ usually minimizes the $M^{2}$ measure.

\section{Conclusions}

The measures $M^{2}$ and $\tilde{N}$ play an important role in the immunization literature. This motivates an extension of the analysis of the theoretical properties and effectiveness of more general dispersion measures. Such alternatives may represent viable options for minimizing the immunization risk. This paper develops a generalized cash flow dispersion measure as an immunization risk measure that includes previously examined quadratic and linear measures. It therefore sets up a tool to be used in empirical testing. Each new measure is derived from a different set of shocks (changes on the term structure of interest rates) and depends on the corresponding subset of worst shocks. 
We show that the set of shocks and worst shocks that give rise to measures between linear and quadratic are the most reasonable. Amongst them, the linear measure has the best properties. The set of shocks that gives rise to the linear measure bounds the variation of the shocks by a constant and has an economic meaning. In that sense, this set is more reasonable than sets of shocks that bound the derivative of the shocks. Furthermore, numerical examples suggest that the linear measure, ceteris paribus, could be the border between the optimal portfolio including a maturity matching bond and a bullet portfolio. Measures lower than the linear one are minimized by portfolios that include a maturity matching bond and measures greater than the linear one by a bullet portfolio. Both portfolios minimize the $\tilde{N}$ linear measure.

For all measures, their associated worst shocks are parallel shifts plus a specific twist of term structure of interest rates around $2 m$. Minimizing each measure minimizes the effect of the worst shock that makes immunization infeasible. We therefore relate these results to factor analysis of the term structure where parallel, steepness and curvature factors are found. The property that worst shocks containing just a parallel shift plus a steepness shift around $2 m$ can be studied empirically and used to design immunized bond portfolios. ${ }^{10}$

Finally, a short empirical study on the Spanish Bond Market in the mid-1990s supports the findings that the portfolio minimizing the linear measure has the best performance and generally includes a maturity matching bond. Furthermore, maturity-constrained duration-matched portfolios, as suggested in Bierwag et al. (1993) and others, both perform well empirically and confirm previous empirical results for US and Canadian markets.

\section{Acknowledgements}

We are grateful to Fritz Laux, Eliseo Navarro, Juan Nave and especially to Gary Anderson and two anonymous referees for many helpful comments on earlier versions of this paper that greatly improved it. We are responsible for any remaining errors. A. Ibáñez is grateful to the College of Business at Arizona State University and to Universitat Pompeu Fabra for their hospitality. DGICYT PB95-0729-C0202 provided partial support for A. Balbás.

\section{Appendix A}

The set $K$ is a subset of the vector space of real-valued functions defined on $[0, T]$. The elements of $K$ could be additive shocks, polynomial shocks, differentiable shocks (as in Fong and Vasicek, 1984), or more general shocks.

\footnotetext{
${ }^{10}$ In related works, Fung and Hsieh (1996) empirically study what they call extreme yield curve event risks and Au and Frishling (1996) also define the maximum potential change of a portfolio.
} 
The $q$ portfolio value at $m$, included the coupons paid before $m$, is given by

$$
V(q, k)=\int_{0}^{T} c(t) \exp \left[\int_{t}^{m}(g(s)+k(s)) \mathrm{d} s\right] \mathrm{d} t
$$

and the price of this portfolio is equal to

$$
C=\int_{0}^{T} c(t) \exp \left[-\int_{0}^{t} g(s) \mathrm{d} s\right] \mathrm{d} t .
$$

Denoting the return of the zero coupon bond with $m$ years to maturity by

$$
R=\exp \left[\int_{0}^{m} g(s) \mathrm{d} s\right]
$$

and the coupon's present value by

$$
c(t, 0)=c(t) \exp \left[-\int_{0}^{t} g(s) \mathrm{d} s\right]
$$

we have

$$
V(q, k)=R \int_{0}^{T} c(t, 0) \exp \left[\int_{t}^{m} k(s) \mathrm{d} s\right] \mathrm{d} t .
$$

The function $V(q, k)$ is convex in $k$, positive, and all the bonds produce the same return under the null shock $(k=0)$.

We will say that a portfolio is immunized for set $K$ if its return is at least $R$, i.e.,

$$
\frac{V(q, k)}{C} \geqslant R \quad \text { for all } k \in K \text {. }
$$

Following the approach of Balbás and Ibáñez (1998), let us now define the set of worst shocks $K_{n}^{*}$ for each $K_{n}, n \in(-\infty,+\infty)$, as those feasible shocks such that any bond looses most value at $m$ when one of these worst shocks occurs. If we observe (A.4), these worst shocks are given by the highest fall of the instantaneous forward interest rate before $m$, and by the highest rise after $m$. Thus, the sets of worst shocks are given by

$$
K_{n}^{*}=\left\{\begin{array}{lr}
\lambda_{0}-\lambda(m-t)^{n-1} & \text { if } 0 \leqslant t<m, \\
\lambda_{0}+\lambda(t-m)^{n-1} & \text { if } m<t \leqslant T, \\
\text { where } \lambda_{0} \text { is any real number }
\end{array}\right\}
$$

for any $n \in N$. The property $K_{n}^{*} \subset K_{n}$ is obvious from (2) and (3).

Lemma 2. For any $n \in(-\infty,+\infty)$ and for any $k \in K_{n}$ there exists $k^{*} \in K_{n}^{*}$ such that

$$
\begin{aligned}
& k^{*}(t) \leqslant k(t) \quad \text { if } 0 \leqslant t<m, \\
& k^{*}(t) \geqslant k(t) \quad \text { if } m<t \leqslant T,
\end{aligned}
$$

where $k^{*}$ is given by (A.5), and if $n>1$ then

$$
\lambda_{0}=k(m)
$$


and if $n \leqslant 1$ then

$$
\begin{aligned}
& \left\{t_{1}, t_{2}\right\}=\arg \min \left\{\lambda\left[\left(t_{2}-m\right)^{n-1}+\left(m-t_{1}\right)^{n-1}\right]-\left[k\left(t_{2}\right)-k\left(t_{1}\right)\right],\right. \\
& \left.0 \leqslant t_{1}<m<t_{2} \leqslant T\right\}, \\
& \lambda_{0}=k\left(t_{2}\right)-\lambda\left(t_{2}-m\right)^{n-1} .
\end{aligned}
$$

Note that from (A.5) and for the significant particular cases $n=1$ and $n=2$, the worst shocks are given by step functions and degree one polynomials, respectively.

Proof of Lemma 2. Consider first the case $n>1$.

If $0 \leqslant t<m$, we have from (2)

$$
k(t) \geqslant k(m)-\lambda(m-t)^{n-1}
$$

and given $k^{*}(t)$ definition in (A.5), then

$$
k(t) \geqslant k^{*}(t)
$$

if $\lambda_{0}=k(m)$.

If $t>m$ we have from (2)

$$
k(t) \leqslant k(m)+\lambda(t-m)^{n-1}
$$

and given (A.5), then

$$
k(t) \leqslant k^{*}(t)
$$

if $\lambda_{0}=k(m)$. Therefore, for $n>1$, (A.6) holds if (A.7) holds.

Consider now the case $n \leqslant 1$, and note that (A.8) provides the values $t_{1}$ and $t_{2}$ $\left(t_{1}<m<t_{2}\right)$ such that (3) is closest to being violated. Once we know $t_{1}$ and $t_{2}, \lambda_{0}$ is given by (A.9) or it could also be defined as $\lambda_{0}=k\left(t_{1}\right)+\lambda\left(m-t_{1}\right)^{n-1}$. Eq. (A.9) guarantees that $k^{*}\left(t_{2}\right)=k\left(t_{2}\right)$, and therefore, that $k^{*}(t) \geqslant k(t)$ if $t>m$ and that $k^{*}(t) \leqslant k(t)$ if $t<m$, since $k^{*}$ verifies (A.5).

If $0 \leqslant t<m$, we know from (A.5)

$$
k^{*}(t)=\lambda_{0}-\lambda(m-t)^{n-1}
$$

and from (3) that

$$
\begin{aligned}
k^{*}(t) & =k\left(t_{2}\right)-\lambda\left(t_{2}-m\right)^{n-1}-\lambda(m-t)^{n-1} \\
& =k(t)-\left[\lambda\left[\left(t_{2}-m\right)^{n-1}+(m-t)^{n-1}\right]-\left[k\left(t_{2}\right)-k(t)\right]\right] \\
& \leqslant k(t)
\end{aligned}
$$

if (A.8) and (A.9) hold.

If $t>m$, we have from (A.5)

$$
k^{*}(t)=\lambda_{0}+\lambda(t-m)^{n-1}
$$


and from (3) that

$$
\begin{aligned}
k^{*}(t)= & k\left(t_{2}\right)-\lambda\left(t_{2}-m\right)^{n-1}+\lambda(t-m)^{n-1} \\
= & k(t)+\left[\lambda\left[(t-m)^{n-1}+\left(m-t_{1}\right)^{n-1}\right]-\left[k(t)-k\left(t_{1}\right)\right]\right] \\
& -\left[\lambda\left[\left(t_{2}-m\right)^{n-1}+\left(m-t_{1}\right)^{n-1}\right]-\left[k\left(t_{2}\right)-k\left(t_{1}\right)\right]\right] \\
\geqslant & k(t)
\end{aligned}
$$

if (A.8) and (A.9) hold. Therefore, for $n \leqslant 1$ (A.6) holds if (A.8) and (A.9) hold.

Proof of Theorem 1. For $n>0,{ }^{11}$ from Lemmas 2 and (A.4), it immediately follows that for any $k \in K_{n}$ there exists a $k^{*} \in K_{n}^{*}$ such that

$$
V(q, k) \geqslant V\left(q, k^{*}\right) \text {. }
$$

Since $V(q, k)$ is Gateaux differentiable with respect to the $k$ variable and noting that a convex functional is always bounded from below by its tangent plane (see Luenberger, 1969), we have that for each $k^{*} \in K_{n}^{*}$,

$$
\begin{aligned}
\frac{V\left(q, k^{*}\right)-R C}{R C} & \geqslant \int_{0}^{T} \frac{c(t, 0)}{C} \int_{t}^{m} k^{*}(s) \mathrm{d} s \mathrm{~d} t \\
& =\int_{0}^{T} \frac{c(t, 0)}{C}\left(\lambda_{0}(m-t)-\frac{\lambda}{n}|t-m|^{n}\right) \mathrm{d} t \\
& =\lambda_{0}(m-D)-\frac{\lambda}{n} M^{n} .
\end{aligned}
$$

\section{References}

Au, K., Frishling, V., 1996. Maximum potential change of a portfolio. Journal of Fixed Income 6 (1), 92-95.

Balbás, A., Ibáñez, A., 1998. When can you immunize a bond portfolio? Journal of Banking and Finance 22 (12), 1571-1595.

Barber, J.R., Copper, M.L., 1998. A minimax risk strategy for portfolio immunization. Insurance: Mathematics and Economics 23, 173-177.

\footnotetext{
${ }^{11}$ For $n=0$ from (3) and $t<m$ we have that $\int_{t}^{m} k^{*}(s) \mathrm{d} s=\int_{t}^{m}\left(\lambda_{0}-\lambda(m-s)^{-1}\right) \mathrm{d} s=\lambda_{0}(m-t)+\left.\lambda \ln (s)\right|_{m-t} ^{0}=\lambda_{0}(m-t)-\infty=-\infty$,
}

and therefore, $(V(q, k)-R C) / R C \geqslant-\infty$. For $t>m$ we also have a bound of $-\infty$. For $n<0$ from (3) and $t<m$ we have that

$$
\int_{t}^{m} k^{*}(s) \mathrm{d} s=\int_{t}^{m}\left(\lambda_{0}-\lambda(m-s)^{n-1}\right) \mathrm{d} s=\lambda_{0}(m-t)+\left.\lambda \frac{s^{n}}{n}\right|_{m-t} ^{0}=\lambda_{0}(m-t)-\infty=-\infty
$$

and therefore, $(V(q, k)-R C) / R C \geqslant-\infty$. For $t>m$ we also have a bound of $-\infty$.

Furthermore, $\exp \left[\int_{t}^{m} k^{*}(s) \mathrm{d} s\right]=\exp [-\infty]=0$ for any $t \neq m$, and consequently, the value of any coupon at $m$ is zero. 
Bowden, R.J., 1997. Generalizing interest rate duration with directional derivatives: Direction $X$ and applications. Management Science 43 (5), 586-595.

Bierwag, G.O., 1977. Immunization, duration, and the term structure of interest rates. Journal of Financial and Quantitative Analysis 12, 725-742.

Bierwag, G.O., Fooladi, I., Roberts, G.S., 1993. Designing an immunized portfolio is $M$-square the key? Journal of Banking and Finance 17, 1147-1170.

Brennan, M.J., Schwartz, E., 1983. Duration, bond pricing and portfolio management. In: Kaufman, G.G. et al. (Eds.), Innovations in Bond Portfolio Management: Duration Analysis and Immunization. JAI Press, Greenwich, CT.

Chambers, D.R., Carleton, W.T., McEnally, R.C., 1988. Immunizing default-free bond portfolios with a duration vector. Journal of Financial and Quantitative Analysis 23 (1), 89-104.

Chance, D.M., Jordan, J.V., 1996. Duration, convexity and time as components of bond returns. Journal of Fixed Income 6 (2), 88-96.

Cox, J.C., Ingersoll, J.E., Ross, S.A., 1979. Duration and the measurement of basis risk. Journal of Business 52, 51-61.

Fisher, L., Weil, R., 1971. Coping with the risk of interest rate fluctuations: Returns to bondholders from naive and optimal strategies. Journal of Business 52, 51-61.

Fong, H.G., Vasicek, O.A., 1984. A risk minimizing strategy for portfolio immunization. Journal of Finance 39 (5), 1541-1546.

Fung, W., Hsieh, D.A., 1996. Global yield curve event risks. Journal of Fixed Income 6 (2), 37-48.

Lacey, N., Nawhalka, S., 1993. Convexity, risk and returns. Journal of Fixed Income 3 (3), 72-79.

Litterman, R., Scheinkman, J.A., 1991. Common factors affecting bond returns. Journal of Fixed Income 54-61.

Luenberger, D.G., 1969. Optimization by Vector Space Methods. Wiley, New York.

Navarro, E., Nave, J.M., 1997. A two-factor model for interest rate risk management. Investigaciones Económicas 21 (1), 55-74.

Prisman, E.Z., Shores, M.R., 1988. Duration measures for specific term structure estimations and applications to bond portfolio immunization. Journal of Banking and Finance 12, 493-504. 